CLINICAL STUDY

\title{
Combined RET and Ki-67 assessment in sporadic medullary thyroid carcinoma: a useful tool for patient risk stratification
}

\author{
Caterina Mian ${ }^{1}$, Gianmaria Pennelli ${ }^{2}$, Susi Barollo ${ }^{3}$, Elisabetta Cavedon ${ }^{1}$, Davide Nacamulli ${ }^{1}$, Federica Vianello ${ }^{3}$, \\ Isabella Negro ${ }^{1}$, Giulia Pozza ${ }^{2}$, Isabella Merante Boschin ${ }^{4}$, Maria Rosa Pelizzo ${ }^{4}$, Massimo Rugge ${ }^{2}$, Franco Mantero ${ }^{1}$, \\ Maria Elisa Girelli ${ }^{1}$ and Giuseppe Opocher ${ }^{3}$ \\ ${ }^{1}$ Endocrinology Unit, Department of Medical and Surgical Sciences, ${ }^{2}$ Pathology Unit, Department of Medical Sciences and Special Therapies, ${ }^{3}$ Veneto \\ Institute of Oncology - IRCCS, Padova and ${ }^{4}$ Special Surgery Unit, Department of Medical and Surgical Sciences, University of Padova, Via Ospedale n. 105 , \\ 35128 Padova, Italy \\ (Correspondence should be addressed to C Mian; Email: caterina.mian@unipd.it)
}

\begin{abstract}
Objective: Medullary thyroid carcinoma (MTC) derives from the parafollicular C cells, being sporadic in $75 \%$ of cases and familial in $25 \%$, due to RET proto-oncogene germinal mutations. In sporadic forms, stage at diagnosis is the most important negative prognostic factor. The aim of this study was to evaluate the prognostic impact of molecular and immunohistochemical markers in sporadic MTC. Design and methods: We studied 60 patients with sporadic MTC. For each case, we sought RET somatic mutations in the primary cancer and in lymph node metastases. The primary cancer also underwent immunohistochemical examination for Ki-67.

Results: A somatic RET mutation was found in 38\% of patients, being M918T in 52\% of them. We observed a statistically significant association between RET mutations and male gender $(P<0.01)$, tumor size $(P<0.05)$, lymph nodes $(P<0.05)$ and distant metastases $(P<0.001)$, advanced stage $(P<0.05)$, increased risk of persistent disease $(P=0.01)$, and low overall survival $(P<0.01)$. High Ki-67 levels were similarly associated with extra-thyroid spread $(P<0.05)$, lymph nodes $(P<0.05)$ and distant metastases $(P<0.001)$, advanced stage $(P=0.01)$, and low overall survival $(P=0.01)$. Combining somatic RET analysis with Ki-67 assessment seems to be useful for increasing the specificity of Ki-67 assessment alone and identifying patients with a more aggressive cancer: in our series, only the patients who died during the follow-up had both a somatic RET mutation and a Ki-67 expression level $>50$ cells $/ \mathrm{mm}^{2}$.

Conclusions: The combined evaluation of RET and Ki-67 could act as an adjuvant prognostic marker useful for ameliorating the initial risk stratification of patients with sporadic MTC.
\end{abstract}

European Journal of Endocrinology $164971-976$

\section{Introduction}

Medullary thyroid carcinoma (MTC) is a rare neuroendocrine cancer originating from parafollicular $\mathrm{C}$ cells producing calcitonin $(\mathrm{Ct})$; it accounts for $5-10 \%$ of all thyroid carcinomas. About $75 \%$ of MTC are sporadic, whereas the remaining are hereditary due to germinal mutations that activate the RET proto-oncogene (1).

Total surgical resection is the only effective therapy for MTC. In a far from negligible number of cases, however, the initial diagnosis occurs late, when the cancer has spread beyond the thyroid bed to loco-regional or even distant sites, so only $50 \%$ of cancer patients can achieve a biochemical cure after surgery $(2,3)$.

Age at diagnosis and disease stage at the time of initial treatment currently represent the main prognostic indicators $(2,4)$. Although clinical and pathological variables alone may suffice to predict patient outcome in most cases, the variable behavior of MTC in some cases suggests that, in the same clinical and pathological conditions, cancer progression and survival may also be influenced by other biological elements $(5,6)$.

In times of molecular analysis, various genetic factors have been shown to play a part in the risk stratification of MTC patients (7-9). In particular, somatic RET mutations and/or RET polymorphisms were analyzed in several series of sporadic MTC, and RET status was found to correlate with patient outcome (10-12). Recent reports have demonstrated that somatic RET mutations occur in 30-50\% of sporadic cases of MTC, and they are a negative predictor of cancer remission and survival (13-15). Such reports were not always homogeneous in terms of the frequency, type, and site of somatic RET mutations, or their association with clinical and pathological features, suggesting that other 
biological markers could also be useful for ascertaining a patient's risk. Given this scenario, a previous study conducted on a small series of familial and sporadic MTC patients with a long follow-up showed that a high $\mathrm{Ki}-67$ expression can predict patient survival independently from other classic prognostic factors (16).

In this study, we combined somatic RET mutation analysis in paired primary MTC and metastatic lymph nodes with the immunohistochemical expression of Ki-67 in a large series of sporadic MTC patients followed up at our institution to ascertain whether the two molecular markers are related to one another and whether they can be used in combination to better characterize the clinical course of sporadic MTC.

\section{Materials and methods}

\section{Patients}

We studied 60 sporadic MTC patients (29 males and 31 females, median age 59, range 22-87 years) with a median follow-up of 39 months (range 6-226 months). All patients underwent total thyroidectomy and central neck compartment dissection, and unilateral or bilateral neck dissection was also performed in 31 cases. Tumors were staged according to the 6th TNM classification (17): 18 were stage I, ten were stage II, ten were stage III, and 22 were stage IV. At initial diagnosis, $48 \%(29 / 60)$ of the patients had an MTC with no evidence of lymph node or distant metastases; $52 \%$ $(31 / 60)$ had cervical lymph node metastases, and ten of them had distant metastases too. Patients were considered biochemically cured on the basis of a $\mathrm{Ct}$ value below $10 \mathrm{pg} / \mathrm{ml} 1$ year after primary surgery and/or at the latest follow-up. At the latest clinical examination, $57 \%(34 / 60)$ of the patients were considered biochemically cured, four patients died of progressive disease during the follow-up.

All patients were assumed to have a sporadic cancer because no germline RET mutations were found, their family history was negative, and no other endocrine neoplasia was identified.

\section{RET analysis}

All patients gave their informed consent to genetic testing. All primary MTCs were collected after surgery, immediately frozen in liquid nitrogen, and stored at $-80^{\circ} \mathrm{C}$. DNA was extracted from primary cancers using the DNeasy Blood and Tissue Handbook (Qiagen). For any metastatic lymph nodes available for genetic testing, DNA was extracted from ten sections each $5 \mu \mathrm{m}$ thick of paraffin-embedded archived material using the same extraction kit. RET exon 10, 11, 13, 14, 15 , and 16 germline mutations were assessed by direct sequencing.

\section{Ki-67 immunostaining and Ki-67 scores}

Immunohistochemical analysis was performed on paraffin-embedded tissue samples, in formalin-fixed sections $5 \mu \mathrm{m}$ thick, using a standardized avidin-biotin complex method in an automatic system (Benchmark XT; Ventana, Tucson, AZ, USA) with CONFIRM anti Ki-67 antibody (Rabbit Monoclonal, clone 30-9 Roche). For the assessment of Ki-67 staining, nuclei were considered positive when the intensity of immunostaining ranged from weak to strong and when positivity was confined to the nucleoli; nuclei with no immunostaining were considered negative. For each patient, a $5 \mathrm{~mm}^{2}$ area of neoplastic tissue was analyzed (corresponding to three random $10 \times$ fields of view, FOV). The number of positive nuclei in the FOV was recorded using a videoanalytical method (the Image-Pro-Plus 7 analysis program) and the final result was reported as the number of positive nuclei per $1 \mathrm{~mm}^{2}$ of neoplastic area. Ki-67 immunostaining was not quantifiable in six patients, one of whom died during the follow-up.

\section{Statistical analysis}

The Kolmogorov-Smirnov test was used to assess the distribution of the different variables: age showed a normal distribution, whereas tumor size and Ki-67 values did not. The $\chi^{2}$ and Mann-Whitney $U$ tests were used according to the variable considered.

For Ki-67 values, we used receiver operating characteristic (ROC) curve analysis to define different cutoffs showing the best sensitivity and specificity for each outcome variable. Then, we calculated the likelihood ratio (with 95\% confidence interval (CI)) to obtain the post-test probability for different cutoffs.

The Kaplan-Meier method and standard log-rank tests were used to evaluate the effect of RET mutations and a done Ki- 67 cutoff on the probability of a patient's survival. The multiple logistic regression test was used to determine the independent effect of somatic RET mutation and Ki-67 scores on the outcome of MTC patients. A $P<0.05$ was considered statistically significant.

\section{Results}

\section{RET analysis and genotype-phenotype correlations}

A total of 20 patients carried a somatic RET mutation in their primary cancer. Paired primary and metastatic MTCs were analyzed in 24 cases: the genotype was concordant in all but three patients whose primary cancer harbored a wild-type RET, while the RET was mutated in the corresponding node metastasis. Overall, $38 \%(23 / 60)$ of patients carried a somatic RET mutation in the primary and/or metastatic MTC. The majority of RET mutations was located in the exon 16 at 
codon 918 (M918T in 12/23, 52\%): this is also the only mutation to be seen, as a de novo event, in the metastatic nodes. Of the 11 patients, four patients carried somatic RET mutations in the exon 10, at codons 618 (C618R, C618S, and C618Y) and 611 (C611R); four patients carried somatic RET mutations in the exon 11 at codons 634 (C634W and C634Y) and 630 (C630R); two patients carried an RET mutation in the exon 15: one was at codon 883 (A883F), whereas the other showed a 12 bp deletion starting from codon 898; and one patient concurrently carried two mutations: one in the exon 16 (M918T) and the other in the exon 13, codon 781 (Q781R). All the patients who died during the follow-up carried a somatic RET mutation.

As shown in Table 1, somatic RET mutations were significantly more frequent in cases of male gender $(P<0.01)$, young age $(P<0.05)$, larger tumors $(P<0.05)$, in the presence of node $(P<0.05)$ and distant metastases $(P<0.001)$, and more advanced stages $(P<0.05)$. Considering the patient's outcome, somatic RET mutations were statistically associated with persistent disease: only $35 \%$ of patients with somatic RET mutations were biochemically cured at the latest follow-up, as opposed to $70 \%$ of the RET-negative cases $(P=0.01$; Table 1$)$. When the survival curves of MTC patients with and without somatic RET mutations were analyzed, the survival curve was significantly lower for patients positive for RET mutations $(P<0.01)$, as shown in Fig. 1.

\section{Ki-67 immunohistochemistry}

Nuclear Ki-67 expression levels were not significantly associated with gender, age, or cancer size.

Using Mann-Whitney $U$ test, it was found that Ki-67 expression levels were significantly higher in MTC with extra-thyroid spread than in those remaining confined to the thyroid $(P<0.05)$, in the presence of node $(P<0.05)$ or distant metastases $(P<0.001)$, in advanced stages $(P=0.01)$, and in patients with persistent disease $(P<0.05)$.

\section{Combined Ki-67 and RET analysis in relation to patient outcome}

On ROC analysis, significant Ki-67 cutoffs were found for the presence of distant metastases $(P<0.0001)$ and the cancer-related death risk $(P=0.0001)$. In patients with persistent disease, the presence of distant metastases was significantly associated with a Ki-67 cutoff $>25$ cells $/ \mathrm{mm}^{2}$ (with $100 \%$ sensitivity and $81 \%$ specificity) and, for a likelihood ratio of 5, this cutoff was associated with a $43 \%$ post-test probability of distant metastases versus a pre-test probability of $16 \%$. All $7 / 7$ patients with distant metastases in the MTC group associated with a Ki-67>25 cells $/ \mathrm{mm}^{2}$ also carried a somatic RET mutation, although this was only true of $22 \%(2 / 9)$ of patients without distant metastases (Fig. 2a). The risk of cancer-related death

Table 1 Clinical and histological features and follow-up in patients with sporadic medullary thyroid carcinoma with and without somatic RET mutations and according to Ki-67 immunohistochemical score $<$ or $\geq 25$ cells $/ \mathrm{mm}^{2}$.

\begin{tabular}{|c|c|c|c|c|c|c|}
\hline & \multicolumn{3}{|c|}{ RET mutations } & \multicolumn{3}{|c|}{ Ki-67 } \\
\hline & $\begin{array}{l}\text { Negative } \\
(n=37)\end{array}$ & $\begin{array}{l}\text { Positive } \\
(n=23)\end{array}$ & $\boldsymbol{P}^{*}$ & $\begin{array}{c}<25 \text { cells } / \mathrm{mm}^{2} \\
(n=38)\end{array}$ & $\begin{array}{c}\geq 25 \text { cells } / \mathrm{mm}^{2} \\
(n=16)\end{array}$ & $\boldsymbol{P}^{\dagger}$ \\
\hline Age at diagnosis (years; median (range)) & $62(57-66)$ & $53(47-66)$ & $<0.05$ & $63(57-64)$ & $53(44-66)$ & 0.01 \\
\hline Gender $(\mathrm{M} / \mathrm{F})$ & $12 / 25$ & $17 / 6$ & $<0.01$ & $16 / 22$ & $8 / 8$ & NS \\
\hline $\mathrm{T}$ categories & & & $<0.05$ & & & NS \\
\hline $\mathrm{T} 1$ & 17 & 4 & & 16 & 4 & \\
\hline T2 & 8 & 6 & & 10 & 3 & \\
\hline T3 & 10 & 11 & & 11 & 9 & \\
\hline $\mathrm{T} 4$ & 1 & 1 & & 1 & 0 & \\
\hline NA & 1 & 1 & & 0 & 0 & \\
\hline Node metastases & & & $<0.05$ & & & $<0.05$ \\
\hline NO & 22 & 7 & & 23 & 5 & \\
\hline N1 & 15 & 16 & & 15 & 11 & \\
\hline Distant metastases & & & $<0.001$ & & & $<0.0001$ \\
\hline MO & 37 & 13 & & 38 & 9 & \\
\hline M1 & 0 & 10 & & 0 & 7 & \\
\hline Stage & & & $<0.05$ & & & 0.005 \\
\hline 1 & 15 & 3 & & 14 & 3 & \\
\hline II & 7 & 3 & & 10 & 1 & \\
\hline III & 6 & 4 & & 8 & 1 & \\
\hline IV & 9 & 13 & & 6 & 11 & \\
\hline Outcome & & & 0.01 & & & $<0.05$ \\
\hline Cured (34) & 26 & 8 & & 25 & 7 & \\
\hline Not cured (20) & 10 & 10 & & 9 & 6 & \\
\hline Dead & 0 & 4 & & 0 & 3 & \\
\hline NA & 1 & 1 & & 2 & 0 & \\
\hline
\end{tabular}

F, female; M, male; T, tumor; NA, not available; NS, not significant. ${ }^{*}$ and ${ }^{\dagger}$ evaluated by $\chi^{2}$ or Fischer's exact test. 


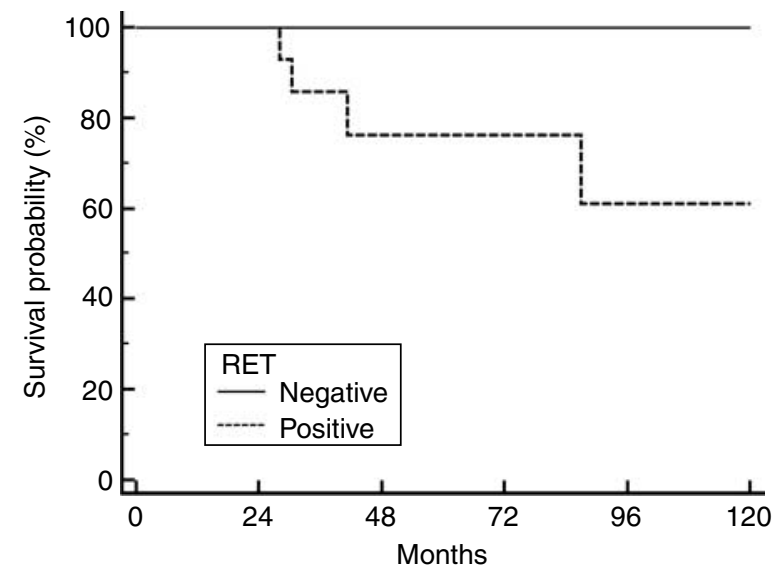

Figure 1 Survival curves for MTC patients with (positive) and without (negative) somatic RET mutations. Patients with somatic RET mutations had a significantly shorter survival $(P<0.01$ by log-rank test).

was significantly associated with a Ki-67 cutoff $>50$ cells $/ \mathrm{mm}^{2}$ (with $100 \%$ sensitivity and $93 \%$ specificity) and, for a likelihood ratio of 15 , it was associated with a $66 \%$ post-test probability of cancerrelated death versus a pre-test probability of only $6 \%$. In the MTC group associated with a Ki-67 > 50 cells $/ \mathrm{mm}^{2}$, only patients carrying an RET somatic mutation died during the follow-up (Fig. 2b). These findings were confirmed on survival curves analysis, where a Ki-67 cutoff $>50$ cells $/ \mathrm{mm}^{2}$ was associated with a lower probability of survival $(P=0.01$; Fig. 3$)$.

When Ki-67 and somatic RET mutations were taken together, the specificity increased from 81 to $95 \%$ for the prediction of distant metastases and from 93 to $100 \%$ for cancer-related death.

As expected, the Mann-Whitney $U$ test showed that patients with somatic RET mutations also carried significantly higher Ki-67 levels than patients without RET mutations (median 25, 95\% CI 13-81 cells $/ \mathrm{mm}^{2}$ versus $13,95 \%$ CI $7-17$ cells $\left./ \mathrm{mm}^{2}, P=0.02\right)$.

Finally, the multivariate analysis, by logistic regression, showed that only advanced stage at diagnosis and somatic RET mutations independently correlated with biochemical cure $(P<0.05)$, while only Ki-67 scores independently correlated with patient cancer-related death $(P<0.0001)$.

\section{Discussion}

In spite of the routine $\mathrm{Ct}$ assessment recommended in patients with thyroid nodes (18), sporadic medullary carcinoma is still often diagnosed only when the cancer has become clinically evident and, in most cases, spread beyond the thyroid, by which time the chances of biochemical cure after surgery are poor, given that stage at initial diagnosis and age are the main prognostic factors $(2,4)$.
In patients who are not biochemically cured, the disease can progress quite rapidly or it may sometimes display a very slow clinical course affording a long patient survival, even in cases with local and/or distant metastases. The initial clinical and pathological findings may therefore not be enough for predicting the future outcome of all cases of MTC.

For the time being, the clinician's only effective tools for gauging the progressive or stable nature of a case of MTC are Ct and carcinoembryonic antigen (CEA) doubling times during the follow-up $(19,20)$. Several studies have attempted to find novel molecular markers capable of identifying the more aggressive MTC with a view to planning appropriate surgery and a tailored patient follow-up right from the start.

In such a scenario, the presence of somatic RET mutations in the primary cancer has been shown to correlate with a worse prognosis in terms of more advanced stage at diagnosis, a higher probability of persistent disease after surgery and a lower survival probability (13-15). A previous study on a small series of primary and metastatic MTC showed that a high Ki-67 expression predicted a shorter survival irrespective of the other classic prognostic factors, but the study
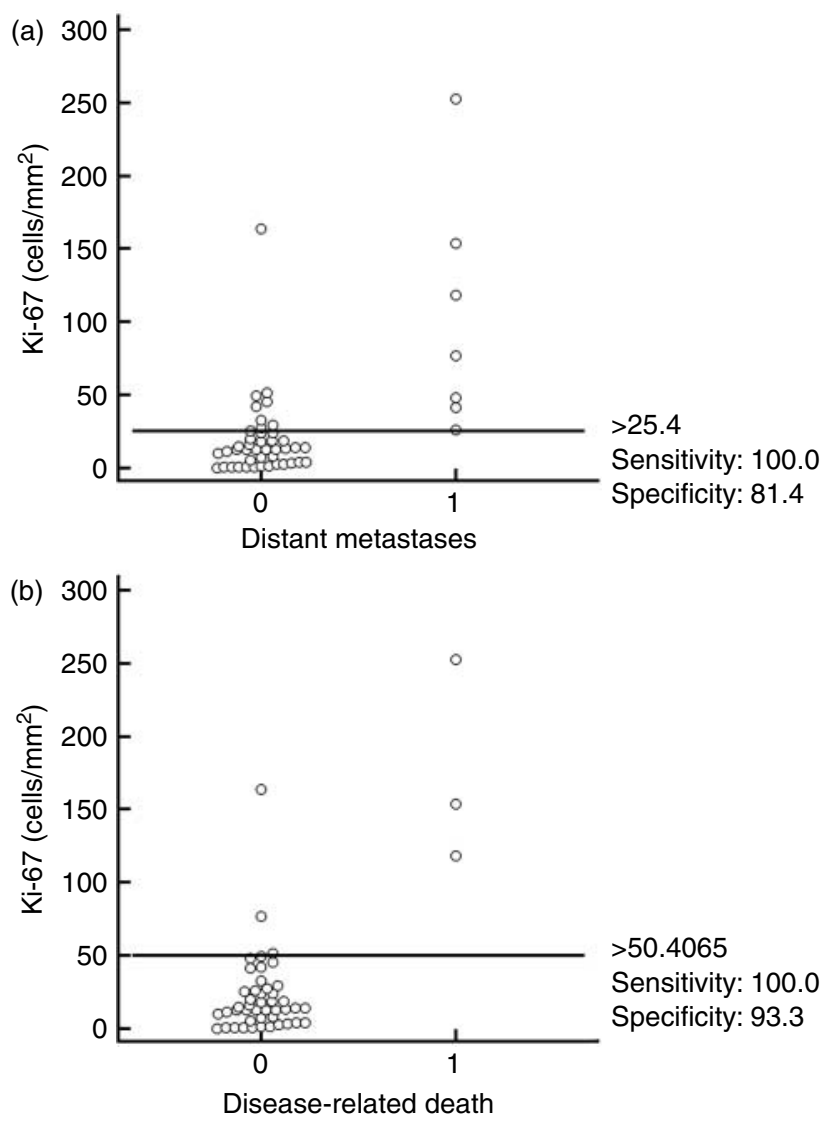

Figure 2 (a) Ki-67 cutoffs, sensitivity, and specificity by ROC curve analysis in MTC patients according to absence/presence (0/1) of distant metastases and (b) survival/disease-related death (0/1) (b). 


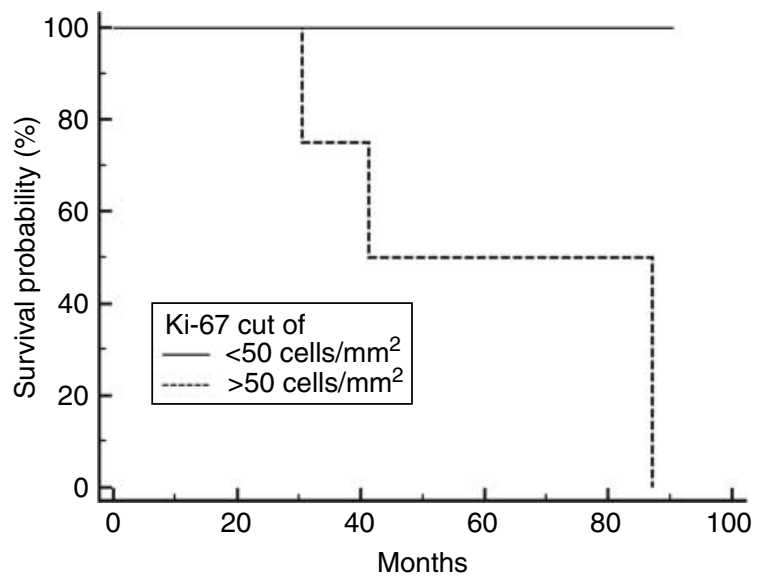

Figure 3 Survival curves for MTC patients with a Ki-67 cutoff $>$ or $<50$ cells $/ \mathrm{mm}^{2}$. Patients with a Ki-67 expression level $>50$ cells $/ \mathrm{mm}^{2}$ showed a significantly shorter survival $(P<0.02$ by log-rank test).

did not consider RET analysis (16). To our knowledge, this study is the first to combine the analysis of somatic RET mutations and Ki-67 expression in primary and metastatic sporadic MTC.

Our RET data confirm previous studies: $38 \%$ of the cases in our series carried somatic RET mutations, 52\% of which were located in the exon 16 , causing the loss of Met at codon 918; we also found (for the first time) that M918T can be acquired as a de novo event in metastatic lymph nodes. Our data confirm that somatic RET mutations correlate with nodal status and distant metastases, a lower probability of achieving biochemical cure after surgery, and a shorter overall survival, indicating that the presence of somatic RET mutations represents a marker of a poor prognosis. In our study, RET mutations were also associated with young age at diagnosis and male gender, a finding reported in only one previous study (13), which may have to do with ethnic or environmental factors, or simply be the result of different patient sampling methods.

Several studies have shown that Ki-67 is of prognostic relevance in human cancers, especially neuroendocrine tumors (21). Only a few studies on small group of patients have addressed Ki-67 analysis (but not RET mutations) in the setting of MTC, with inhomogeneous results. Like Tisell et al., (16) we found that the nuclear expression of Ki-67, combined with RET mutation status, correlated with the clinical course of sporadic MTC, being significantly associated with extra-thyroid spread, advanced stages, progressive disease, and shorter overall survival. More interesting from a practical point of view, we showed that certain Ki-67 cutoffs, i.e. $>25$ and $>50$ cells $/ \mathrm{mm}^{2}$, can predict the presence of distant metastases and the risk of cancerrelated death with a sensitivity of $100 \%$ in both cases, and a specificity of 81 and $93 \%$ respectively. These data are fully consistent with those reported by Faggiano et al. (22) who demonstrated that the MTC proliferative profile is a useful tool for selecting appropriate imaging techniques for persistent MTC patients: in particular, high Ki-67 scores earmarked patients expected to have positive FDG-PET results. In other words, high Ki-67 cutoffs could identify a subset of sporadic MTC cases with an aggressive behavior. Our data also demonstrated that RET findings parallel the results of Ki-67 expression; indeed, mutated RET protein promotes cancer cell proliferation in vitro.

Combining somatic RET analysis with Ki-67 assessment seems to be useful for increasing the specificity of Ki-67 assessment alone and identifying patients with a more aggressive cancer: in our series, only the patients who died during the follow-up had both a somatic RET mutation and a Ki-67 expression level $>50$ cells $/ \mathrm{mm}^{2}$ in their primary cancer. It is likely that the early, possibly pre-surgical, identification of aggressive MTC by such biomarkers adds little to the already available preoperative clinical and radiological setting. Anyway, as it is still debated if prophylactic lateral neck dissection should be performed on principle, in case of clinically apparent MTC (recommendation 61) (3), the identification of cases more at risk to spread to loco-regional lymph nodes could address to the most appropriate surgical strategy, with the aim to increase the probability of cure after surgery. More importantly, in our opinion, the early recognition of the MTC prone to develop progressive disease could lead post-surgical management in biochemically positive patients in terms of defining the timing of subsequent follow-up, planning the right moment for a complete imaging workup to localize metastatic disease, and then selecting the most appropriate radiological technique, such as FDGPET/CT procedures.

In conclusion, our study shows that combined RET and Ki-67 analyses could be useful for the risk stratification of patients with sporadic MTC. Assessing these two molecular markers at the onset, preferably before any initial surgery, may help to orient decisions concerning the extent of initial surgery and the subsequent follow-up.

\section{Declaration of interest}

The authors declare that there is no conflict of interest that could be perceived as prejudicing the impartiality of the research reported.

\section{Funding}

This research did not receive any specific grant from any funding agency in the public, commercial or not-for-profit sector.

\section{Acknowledgements}

We thank Frances Coburn for text editing. We also thank Mr Cristiano Lanza, Dr Luciano Giacomelli, and Mrs Laura Zambonin for their excellent technical support. 


\section{References}

1 Leboulleux S, Baudin E, Travagli J \& Schlumberger M. Medullary thyroid carcinoma. Clinical Endocrinology $200461299-310$. (doi:10.1111/j.1365-2265.2004.02037.x)

2 Gharib H, McConahey WM, Tiegs RD, Bergstralh EJ, Goellner JR, Grant CS, van Heerden JA, Sizemore GW \& Hay ID. Medullary thyroid carcinoma: clinicopathologic features and long-term follow-up of 65 patients treated during 1946 through 1970. Mayo Clinic Proceedings 199267 934-940.

3 Kloos RT, Eng C, Evans DB, Francis GL, Gagel RF, Gharib H, Moley JF, Pacini F, Ringel MD, Schlumberger M \& Wells SA Jr. Medullary thyroid cancer: management guidelines of the American Thyroid Association. Thyroid 200919 565-612. (doi:10.1089/thy.2008.0403)

4 Girelli ME, Nacamulli D, Pelizzo MR, De Vido D, Mian C, Piccolo M \& Busnardo B. Medullary thyroid carcinoma: clinical features and long-term follow-up of seventy-eight patients treated between 1969 and 1986. Thyroid 19988 517-523. (doi:10.1089/thy. 1998.8.517)

5 Dottorini ME, Assi A, Sironi M, Sangalli G, Spreafico G \& Colombo L. Multivariate analysis of patients with medullary thyroid carcinoma. Prognostic significance and impact on treatment of clinical and pathologic variables. Cancer 1996 77 1556-1565. (doi:10.1002/(SICI)1097-0142(19960415)77: $8<1556::$ AID-CNCR20 > 3.0.CO;2-Y)

6 Modigliani E, Cohen R, Campos JM, Conte-Devolx B, Maes B, Boneu A, Schlumberger M, Bigorgne JC, Dumontier P, Leclerc L, Corcuff B \& Guilhem I. Prognostic factors for survival and for biochemical cure in medullary thyroid carcinoma: results in 899 patients. The GETC Study Group. Groupe d'étude des tumeurs à calcitonine. Clinical Endocrinology 199848 265-273. (doi:10. 1046/j.1365-2265.1998.00392.x)

7 Joshi PP, Kulkarni MV, Yu BK, Smith KR, Norton DL, Van Veelen W, Höppener JW \& Franklin DS. Simultaneous downregulation of CDK inhibitors p18(Ink4c) and p27(Kip1) is required for MEN2ARET-mediated mitogenesis. Oncogene 200726 554-570. (doi:10. 1038/sj.onc.1209811)

8 Cavalheiro BG \& Rodrigues JC. Expression of MMP-2 and TIMP-2 in medullary thyroid carcinoma. Thyroid $2008 \mathbf{1 8} 865-871$. (doi:10.1089/thy.2007.0412)

9 Buergy D, Weber T, Maurer GD, Mudduluru G, Medved F, Leupold JH, Brauckhoff M, Post S, Dralle H \& Allgayer H. Urokinase receptor, MMP-1 and MMP-9 are markers to differentiate prognosis, adenoma and carcinoma in thyroid malignancies. International Journal of Cancer 2009125 894-901. (doi:10.1002/ ijc.24462)

10 Uchino S, Noguchi S, Yamashita H, Sato M, Adachi M, Watanabe S, Ohshima A, Mitsuyama S, Iwashita T \& Takahashi M. Somatic mutations in RET exons 12 and 15 in sporadic medullary thyroid carcinomas: different spectrum of mutations in sporadic type from hereditary type. Japanese Journal of Cancer Research 199990 1231-1237.

11 Schilling T, Bürck J, Sinn HP, Clemens A, Otto HF, Höppner W, Herfarth C, Ziegler R, Schwab M \& Raue F. Prognostic value of codon 918 (ATG $\rightarrow$ ACG) RET proto-oncogene mutations in sporadic medullary thyroid carcinoma. International Journal of Cancer 200195 62-66. (doi:10.1002/1097-0215(20010120) 95:1<62::AID-IJC1011>3.0.CO;2-1)
12 Fugazzola L, Muzza M, Mian C, Cordella D, Barollo S, Alberti L, Cirello V, Dazzi D, Girelli ME, Opocher G, Beck-Peccoz P \& Persani L. RET genotypes in sporadic medullary thyroid cancer: studies in a large Italian series. Clinical Endocrinology 200869 418-425. (doi:10.1111/j.1365-2265.2008.03218.x)

13 Dvorakova S, Vaclavikova E, Sykorova V, Vcelak J, Novak Z, Duskova J, Ryska A, Laco J, Cap J, Kodetova D, Kodet R, Krskova L, Vlcek P, Astl J, Vesely D \& Bendlova B. Somatic mutations in the RET proto-oncogene in sporadic medullary thyroid carcinomas. Molecular and Cellular Endocrinology 2008284 21-27. (doi:10. 1016/j.mce.2007.12.016)

14 Elisei R, Cosci B, Romei C, Bottici V, Renzini G, Molinaro E, Agate L, Vivaldi A, Faviana P, Basolo F, Miccoli P, Berti P, Pacini F \& Pinchera A. Prognostic significance of somatic RET oncogene mutations in sporadic medullary thyroid cancer: a 10-year followup study. Journal of Clinical Endocrinology and Metabolism 200893 682-687. (doi:10.1210/jc.2007-1714)

15 Moura MM, Cavaco BM, Pinto AE, Domingues R, Santos JR, Cid MO, Bugalho MJ \& Leite V. Correlation of RET somatic mutations with clinicopathological features in sporadic medullary thyroid carcinomas. British Journal of Cancer $2009 \mathbf{1 0 0}$ 1777-1783. (doi:10.1038/sj.bjc.6605056)

16 Tisell LE, Oden A, Muth A, Altiparmak G, Mõlne J, Ahlman H \& Nilsson 0 . The Ki67 index a prognostic marker in medullary thyroid carcinoma. British Journal of Cancer 200389 2093-2097. (doi:10.1038/sj.bjc.6601453)

17 American Joint Committee on Cancer. The thyroid gland. In AJCC Cancer Staging Manual, 6th edn., pp 77-87. New York: WileyLiss, 2002.

18 Elisei R. Routine serum calcitonin measurement in the evaluation of thyroid nodules. Best Practice and Research. Clinical Endocrinology and Metabolism 200822 941-953. (doi:10.1016/j.beem.2008. 09.008)

19 Giraudet AL, Al Ghulzan A, Aupérin A, Leboulleux S, Chehboun A, Troalen F, Dromain F, Lumbroso J. Baudin E \& Schlumberger M. Progression of medullary thyroid carcinoma: assessment with calcitonin and carcinoembryonic antigen doubling times. European Journal of Endocrinology $2008 \mathbf{1 5 8}$ 239-246. (doi:10.1530/EJE-07-0667)

20 Meijer JA, Le Cessie S, van den Hout WB, Kievit J, Schoones JW, Romijn JA \& Smit JW. Calcitonin and carcinoembryonic antigen doubling times as prognostic factors in medullary thyroid carcinoma: a structured meta-analysis. Clinical Endocrinology 201072 534-542. (doi:10.1111/j.1365-2265.2009.03666.x)

21 Rugge M, Fassan M, Clemente R, Rizzardi G, Giacomelli L, Pennelli G, Mescoli C, Segat D \& Rea F. Bronchopulmonary carcinoid: phenotype and long-term outcome in a singleinstitution series of Italian patients. Clinical Cancer Research 200814 149-154. (doi:10.1158/1078-0432.CCR-07-1631)

22 Faggiano A, Grimaldi F, Pezzullo L, Chiofalo MG, Caracò C, Mozzillo N, Angeletti G, Santeusanio F, Lombardi G, Colao A, Avenia N \& Ferolla P. Secretive and proliferative tumor profile helps to select the best imaging technique to identify postoperative persistent or relapsing medullary thyroid cancer. Endocrine-Related Cancer 200916 225-231. (doi:10.1677/ERC-08-0152)

Received 14 March 2011

Accepted 21 March 2011 